\title{
Mechanism of Occurrence of Toxemia of Late Pregnancy
}

By

\author{
Katsuji Kushima \\ From the Department of Obstetrics and Gynecology, Tohoku \\ University School of Medicine, Sendai
}

(Received for publication, December 15, 1960)

\section{INTRODUCTION}

There have been many theories propounded in the past concerning late pregnancy toxemia, as well as the reports on experimental toxemia in animals that were undertaken to enclose them. At present, however, we know of no satisfactory theory on the genesis of the toxemia.

This toxemia is a syndrome, and has various histological changes in many organs.

Accordingly, the present author felt justified in assuming that, if a theory on the toxemia is sound, it must be capable of consistently explaining all these changes, and if an experimental toxemia is a faithful reproduction of the toxemia in human patients, the method used for inducing it or a closely related method should be capable of causing all these pathological symptoms and changes.

The present author and his co-workers have been engaged for these 18 years in searching for such a theory on late pregnancy toxemia and a method of experimentally inducing it, as would fully satisfy the above conditions. We will report in summary on our studies in the following.

\section{EXPERIMENTAL METHODS}

a) Stimulation of hypothalamus (K. Kushima)

Taking up the hint furnished by the similarity of many symptoms in toxemia with as many symptoms in diencephalic syndrome we experimented with stimulating the nucleus ventromedialis hypothalamicus of rabbit and succeeded in inducing the toxemia-like changes as listed below. According to the researches of $\mathrm{T}$. Kurotsu'), the point of an injecting syringe perpendicularly inserted into the cranium of rabbit to the depth of $15.5 \mathrm{~mm}$ at a point $1.0-2.0 \mathrm{~mm}$ lateralwards from the intersection of the suturae sagittalis and coronalis will reach the $\mathrm{N}$. ventromedialis. Through a syringe thus inserted, we introduced a drop (ca. $0.15 \mathrm{VU}$ ) of neurohypophyseal hormone - the hormone that is found particularly

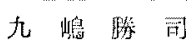


increased in late pregnancy: Yamada, one of the co-workers, has reported on the increase of this hormone in the diencephalon of pregnant rabbits over the content in that of non-pregnant rabbits, and also found a heightened activity of pitutrin-like substance in the blood sampled from the vena uterina coming from a pregnant uterus. S. Akiyama, one of the co-workers, found the Gomoriphil substance in the hypothalamus of rats markedly increased following transplantation of a slice of placenta beneath the dorsal skin. Accordingly, we may presume that the neurohypophyseal hormone may serve as a stimulant of the hypothalamus.

b) Intravenous injection of placental substances

For this purpose, we used Schneider's thromboplastin ${ }^{2}$, Magara's polypeptide ${ }^{3)}$, Kaku's polysaccaride" ${ }^{4}$ and also 10 varieties of substances separately extracted from normal and toxemic placentae, respectively, by $\mathrm{H}$. Yoshizaki ${ }^{5}$, one of our co-workers (Tab. II). These were intravenously administered to rats.

Table I. A List of Symptoms and Changes in Late Pregnancy Toxemia

\begin{tabular}{|c|c|}
\hline Clinical symptoms & $\begin{array}{l}\text { Cardinal symptoms }\left\{\begin{array}{l}\text { edema } \\
\text { proteinuria } \\
\text { hypertension }\end{array}\right. \\
\text { Special toxemias }\left\{\begin{array}{l}\text { eclampsia } \\
\text { abruptio placentae } \\
\text { toxemic pulmonar edema } \\
\text { toxemic cerebral apoplexia } \\
\text { anemia (hydremia) } \\
\text { retinopathia toxemica }\end{array}\right.\end{array}$ \\
\hline Injury of organs & 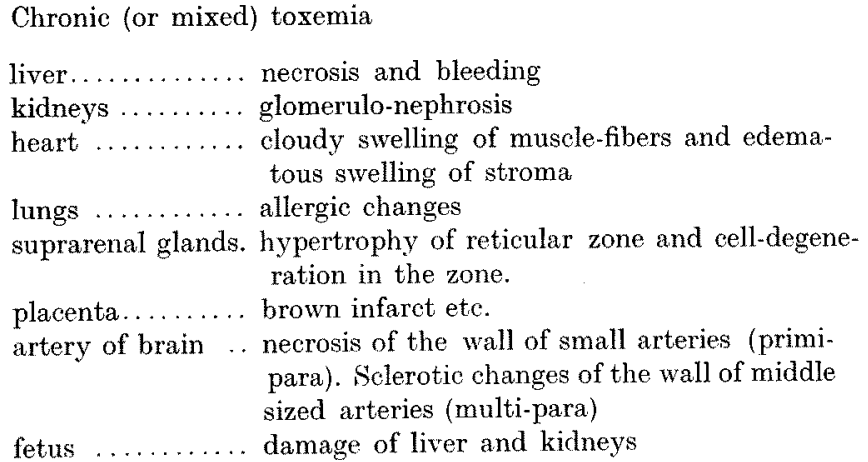 \\
\hline
\end{tabular}

Our Experimental Toxemia

a) Albuminuria (Co-workers: R. Kikuchi and Y. Kato)

The above method perfected by Kushima was applied in experiment with 18 rabbits, of which 16 excreted $0.2-2.0 \%$ of albumin in the urine. The albumin content rose to the maximum in $2-4 \mathrm{hrs}$, then dropped off and became negative 
TABLE II. Isolation Process of Placental Components

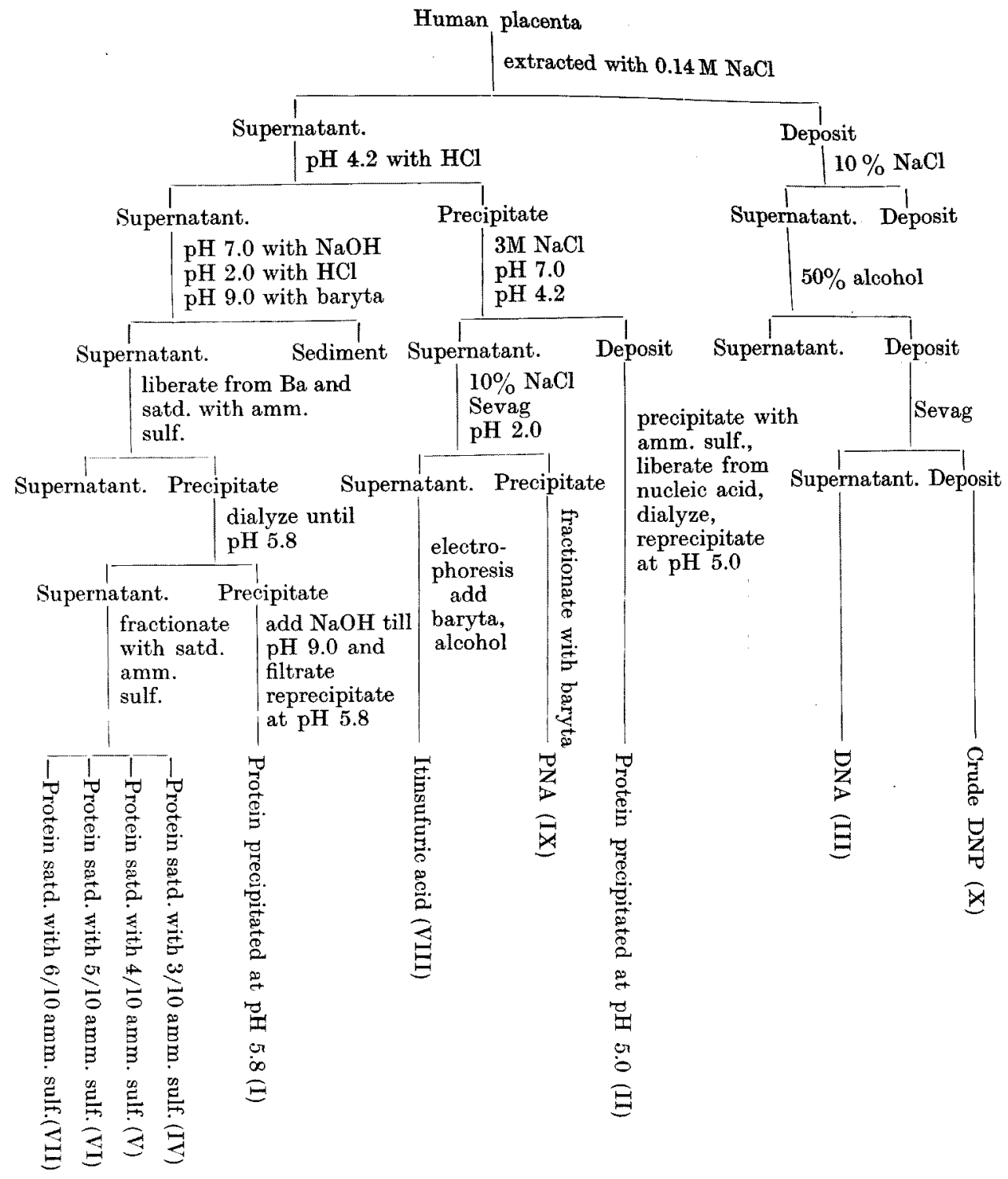

in 24 hrs. after the injection of neurohypophyseal hormone. When the animals were pretreated with subcutaneous injection of phenobarbital prior to the hormone injection, the albuminuria was perceptibly inhibited. When Ringer's solution was injected instead of neurohypophyseal hormone, the albuminuria was also milder.

The animals were sacrificed while the albuminuria was positive, while no 


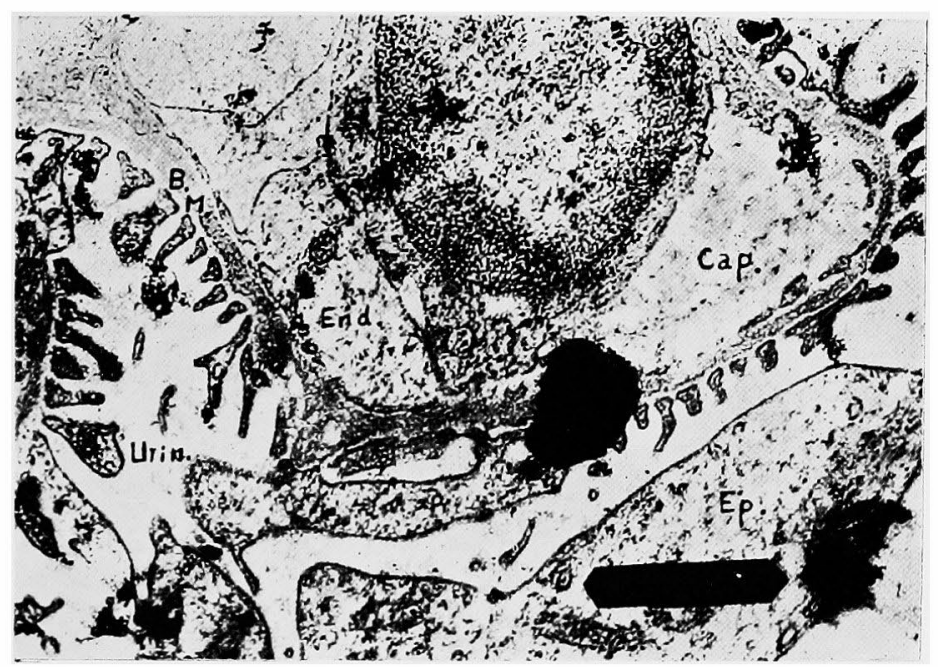

Fig. 1. Glomerular capillary wall of rabbit's kidney without proteinuria.

End: Endothelial cell, Ep: Epithelial cell, B.M.: Basement membrane, R.B.C.: Erythrocytes, Urin: Urinæry space Cap: Capillary space

albuminuria was induced and when the albuminuria had disappeared $24 \mathrm{hrs}$ after the hormone injection, and their kidneys were examined under an electron microscope. The basement membrane of the capillaries of the renal glomeruli was found thickened and showing lowered electron density while the albuminuria was positive, but normal in the cases with negative albuminuria (Figs. 1 and 2). That is to say, the fine structure of the glomeruli was found changing in parallel with the grade of albuminuria.

\section{b) Hypertension (Co-worker: F. Narita)}

Assali claims that toxemic hypertension is induced by humoral factors and cannot be suppressed with sympathetic blockers, but clinical experiences have revealed that such blockers are effective in relieving hypertension in some toxemic cases and not in the other, that is, some of the cases have nervous hypertonia and the others humoral hypertension. In the animals with experimental toxemia induced by Kushima's method, a rise of blocd pressume, by $32 \mathrm{~mm} \mathrm{Hg}$ at the maximum, was always present, though the rate of rise showed individual difference. This hypertension seems to be due to neural factors.

c) Edema (Co-workers: M. Tanaka, F. Shigeno, K. Hagane and R. Komukai)

With the aim of testing the water affinity of the skin, reflecting the edematous tendency, urticae were formed by intradermal injection of physiological saline and the time of absorption was measured. The absorption time was found 


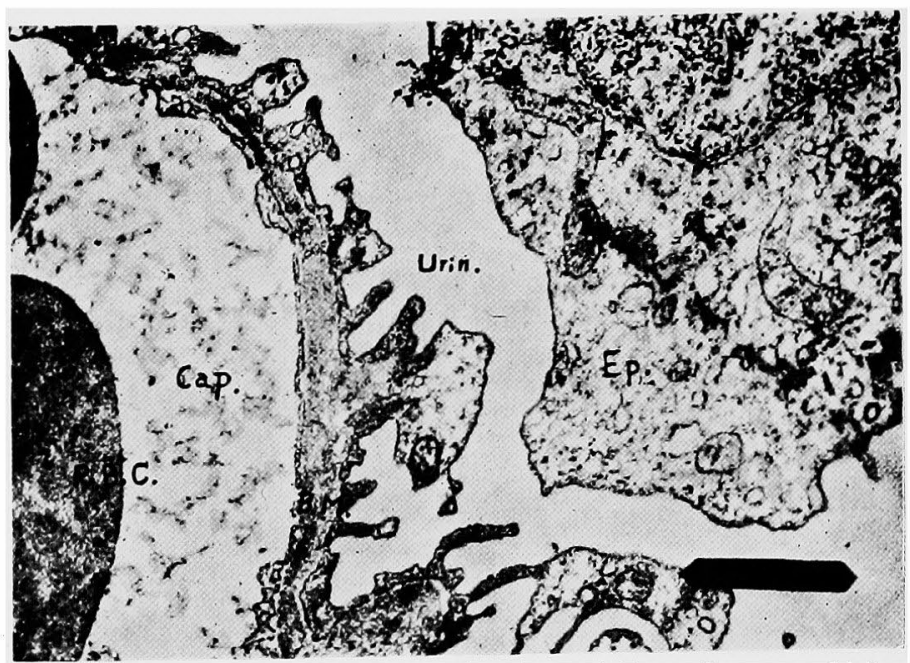

Fig. 2-a Glomerular capillary wall of the kidney from a rabbit with marked proteinuria, two hours after the injection.

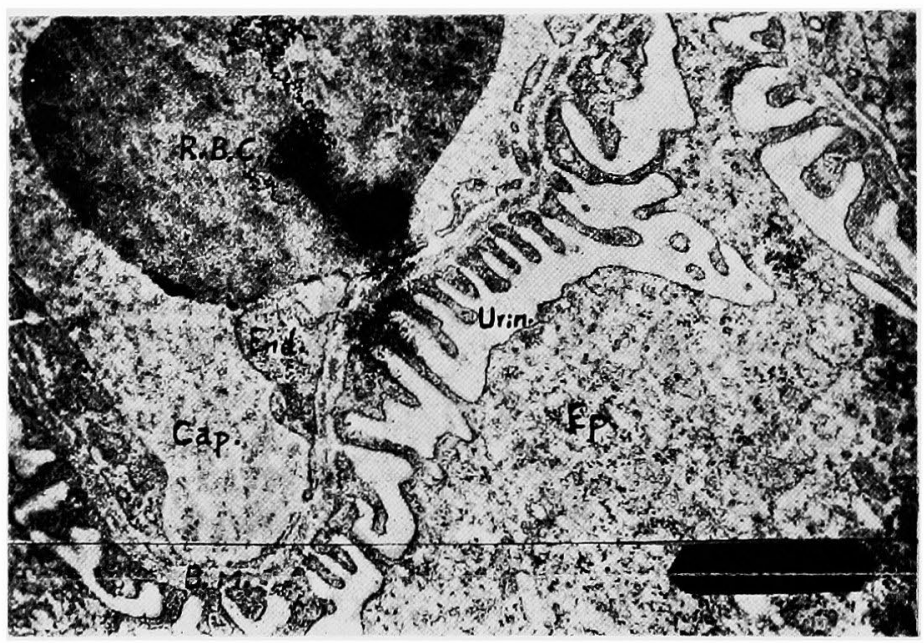

Fig. 2-b Glomerular capillary wall of the kidney from a rabbit twentyfour hours after the injection. (When proteinuria disappeared)

(The scale in each figure indicates $1 \mu$ length.)

shortened 60 min. after stimulation of the hypothalamus, indicating a strengthened tendency to edema.

By measuring the potential generated by insertion of chlorine electrodes beneath the sking of rabbit with a vacuum potentiometer, the potential proportionate to the $\mathrm{Cl}$ concentration was found gradually rising after stimulation of hypothalamus. As it is presumable that the $\mathrm{Cl}$ content in tissues migrates 
in combination with sodium, this finding also suggests a strengthening of the tendency to edema.

d) Eclampsia (Co-worker : H. Tani)

No convulsion could be induced by a single injection of a drop of neurohypophyseal hormone into the hypothalamus, but when the injection was daily repeated, eclampsia-like convulsions were caused after the 3 rd or 4 th round in about half of the cases.

e) Abruptio placentae (Co-worker: C. Nakazato)

A review of the past reports on successful experiments of inducing apoplexia uteroplacentae in animals raises doubts about the true nature of the results whether an abruptio or an abortion is represented in most cases. Therefore, we sought for some symptoms besides ablation of the placenta characterizing this trouble in 10 human uteri of uteroplacental apoplexy cases, and found bleeding and edema, sometimes rupture of small veins, in the myometrium to be specific to such apoplexy. The fibrinous necrosis of the artery walls and fibrinoid thrombosis as cited in the past reports were indeed observed in our study too, but we did not think it probable that these are the cause of the trouble.

When the nervus splanchnicus of pregnant rabbits was electrically stimulated, in accordance with the method of experiment by Couvelaire ${ }^{6}$, edema was caused in the myometrium, but no hemorrhage. Therefore, we inferred that there must be some additional factor for actual occurrence of abruptio and considered the factor of sclerosis of the arteries, as frequently obstrved in human abruptio placentae, in addition to the direct cause. Thus, 0.5 cc each of $0.1 \%$ epinephrine was subcutaneously injected for 10 consecutive days and then the N. splanchnicus was stimulated. This caused occurrence of obvious abruptiolike changes. When injection of small doses of neurohypophyseal hormone into the nucleus ventromedialis hypothalamicus is kept up for many days, for the purpose of stimulating the center of sympathetic nervous system, similar abruptiolike changes were induced. In this case too, a preceding injection of epinephrine continued for some days would intensify the changes induced.

From such experimental results, it may be deduced that abruptio placentae. is caused by excessive stimulation of the sympathetic nerve, that is, it is a Reilly's phenomenon. Such an excessive stimulation may be caused by an increase of the neurohypophyseal hormone content in the diencephalon or a release of some placental substance, as stated below, into the blood. Abruptio may follow a mere stimulation of the nerve center, if it is strong enough, but if arteriosclerosis preexists, it may be more readily induced.

f) Toxemic pulmonary edema (Co-worker: J. Nishi)

Only Vaglio ${ }^{7}$ has reported on successful production of experimental 
toxemic pulmonary edema. He says that intravenous injection of epinephrine preceded by injection of gonadotrophin induces such a change, but our followup experiment failed in obtaining the result. We injected 0.14-0.25 VU per $\mathrm{kg}$ of body weight of neurohypophyseal hormone into the cisterna of rabbits and $0.085-0.25 \mathrm{mg} / \mathrm{kg}$ of epinephrine $5-10 \mathrm{~min}$. after it, and severe pulmonary edema could be induced. Use of Ringer's solution instead of the hormone or mere injection of the hormone into the cisterna alone caused no pulmorary edema. It has been ascertained that a part of the neurohypophyseal hormone secreted in the diencephalon is transported to the third ventricle. This means that when a hypersecretion of the hormone occurs in the hypothalamus, the content of the homone in the ventricle also increases, and a preparatory stage for pulmonary edema to occur is established. Injection of epinephrine may be assumed to cause an irritation of the sympathetic nervous system or quasi-emergency reaction. The low frequency of actual occurrence of toxemic pulmonary edema is probably due to the rarity of coexistence of such a preparatory stage, a state like that following epinephrine injection.

\section{g) Toxemic cerebral bleeding (Co-worker: H. Saito)}

Most of cerebral bleeding in toxemia is associated with eclampsia. Histological examination of cerebral arteries of the cases killed by eclampsia plus cerebral hemorrhage, revealed that in the primiparae, the cerebral arterioles were subject to necrosis, while in the multiparae, hypertensive sclerotic changes were the leading findings in the brain arteries. Intravenous injection of cardiazol caused vehement convulsion but no change in the cerebral blood vessels in rabbits. Rabbits with eclampsia-like convulsions induced by daily injection of neurohypophyseal hormone in the nucleus ventromedialis hypothalamicus for sufficiently many days were found to show changes in the cerebral arterioles similar to those observed in human primiparous eclampsia cases. This experimental method, however, failed in causing cerebral vascular changes as observed in multipara eclampsia cases.

h) Changes in the eye fundus (Co-woker: M. Yamada)

The eye fundus, or the retina, of the toxemic cases is subject to contraction of the blood vessels and edema. Only spasms of the retinal blood vessels immediately followed stimulation of the hypothalamus, but in $20 \mathrm{~min}$. or so, the papilla began to swell, obscuring their boundaries, and some other findings indicative of fundus edema also appeared.

i) Functional and histological changes of the liver (Co-worker: K. Nagai)

Upon testing the liver function of diencephalon-stimulated rabbits by brome-.. sutfalein test,- the function was found mildly- or moderately impaired. Changes 
reminding of eclamptic liver were observed in the rabbits with repeatedly stimulated midbrain.

j) Histological changes of the kidney (Co-worker : R. Kikuchi)

Findings indicative of glomerulonephrosis were found mildly in the kidneys of rabbits stimulated hypothalamus.

k) Ditto of the heart (Co-worker : M. Maeno)

Autopsy of cases who had toxemia in life revealed advanced cloudy swelling of the cardiac muscles, atrophy of the muscle fibers and edematous swelling of the stroma. In the animals with experimental toxemia induced by our method, such cardiac changes were found more intense than in animals with experimental toxemia induced by any other method.

1) Ditto of the adrenal cortex (Co-worker: K. Izaka)

Histological examination of the adrenal cortex of the pathologically autopsied cases with and without toxemia in comparison showed that hypertrophy of the glomerular zone, degeneration of the cells and diminution of lipids in that zone were the characteristic findings in the toxemic cases. Such changes were frankly observable in the adrenal cortex of our rabbits with stimulated hypothalamus.

$\mathrm{m})$ Ditto of the liver and the kidneys of the fetus (Co-worker: $\mathrm{S}$. Miyauchi)

It is a well-known fact that changes similar to the toxemic changes observed in the maternal liver and kidneys are reproduced in the same organ of the newborn child.

When 2 or 3 injections of small dosis of neurohypophyseal hormone are administered to pregnant rabbits into the hypothalamus, changes suggestive of eclamptia liver and glomerulonephrosis were observed in the fetus.

n) Allergic changes of tissues (Co-worker: K. Nagai and K. Sasano)

Many authors claim the nature of toxemia to be allergic, because of the presence of allergic changes in various organs in the affected cases as the ground of their claim. In our animals with experimental toxemia induced by means of stimulation of the hypothalamus, there is no chance for occurrence of antigenantibody reaction. Nonetheless, the liver of rabbits with neurohypophyseal hormone instilled in the hypothalamus showed allergic changes, including edematous swelling of the connective tissue in the Glisson's capsules, infiltration of round cells and histiocyte-like cells into them, thickening and swelling of the arterial walls, proliferation of the nuclei in the arterial intima and hematostasis and fibrin-thrombi in the portal vein. Such allergic changes were present also in 
the lung. Accordingly, we may affirm the resemblance of our experimental toxemia to the human toxemia in respect of allergic findings too.

o) Histological and electron-microscopic changes in the placenta(Co-worker: (K. Noda, I. Noji and K. Aneha)

Our investigation revealed that brown infarct is an item specific to human toxemia. White infarct, syncitium-cell nodes, mixed figure of anaemic and hyperaemic villi, fibrin-thrombi in the chorion, capillarectasia or capillary contraction, haematomata in the intervillous spaces, fibrinoid deposits on the villi or the decidua, hemorrhage in the decidua and the placental stroma, necrosis and hypertrophy of the vascular walls, infiltration of small round cells into the stroma and edematous change of the placental septa could be more frequently detected in toxemic than in normal placenta. Such toxemic placental changes were evoked very perceptibly upon stimulation of the hypothalamus. Considering such findings, we may infer that, instead of primary occurrence of placental changes causing toxemia, anomalies are first induced in the midbrain, involving occurrence of changes in the placenta, which in turn entail production of the substances that partake in the genesis of toxemia.

It has been the accepted opinion that infarct of the placenta is caused by necrosis due to disorders in the blood flow to the peripheral part of the blood vessels following spasms of blood vessels in the villi. But upon closer examination, it was found that sometimes the infarcted villi contain fresh erythrocytes, and sometimes are covered by deposited fibrin. These findings seem to suggest that the anomalies produced in the maternal-side vascular system entails changes in the blood filling the intervillous spaces, causing precipitation of fibrin on the villi, which are thereby hampered in their alimentary ingestion and finally fall a prey to infarct.

Upon examination of normal placenta and placentae sampled from acute and chronic (mixed) toxemia cases under an electron microscope, changes specific to toxemic placentae were detected. Whereas only a small number of endoplasmic reticula could be found in the syncytial cells of normal placentae, innumerable endoplasmic reticula could be observed in those of toxemic placentae, giving a honeycomb-like appearance to the cells. In acute toxemia, the vacuoles are of nearly uniform size, but in chronic toxemic these were of varying sizes and the whole appearance reminded of a cystoglandular hyperplasia of the endometrium (Figs. 3. 4.5). Similar findings were observable when intermittent anoxia was induced in the placenta or the hypothalamus was stimulated in our experimental animals.

p) Other changes

Anaemia in toxemia and chronic toxemia could not be induced by our experimental method of stimulating the hypothalamus. 


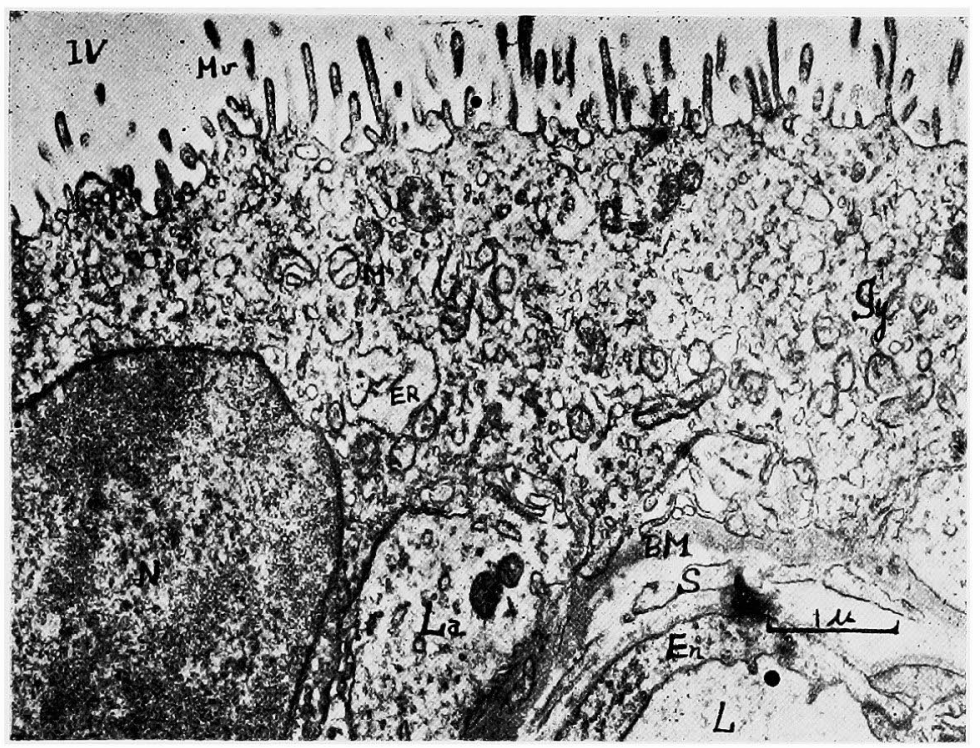

Fig. 3. A chorionic epithelium-cell in a placenta from normal gravida of 10 months of pregnancy. $(\times 5,000)$

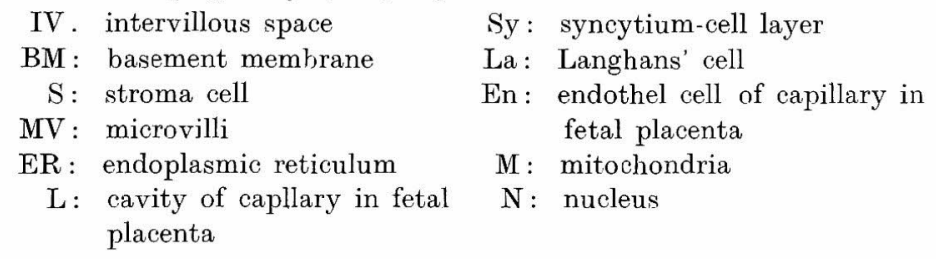

\section{Toxemia and Placental Components}

The placental components isolated from normal human placentae consisting in Magara's substance (polypeptide), Kaku's substance (polysaccharide) and thromboplastin, and 10 substances (Tohoku substances) isolated from normal and toxemic placentae were experimentally tested.

a) Magara's substance

Pregnant rabbits were injected with $20 \mathrm{mg}$ each of PS fraction of Magara's substances. The urinary protein remained negative following the injection in the animals with originally negative protein in urine, but in the animals showing positive urinary protein before the injection, the value was found risen very slightly after it.

Following the injection, the blood pressure always rose, by $32 \mathrm{mmHg}$ at the maximum, but this rise could be totally inhibited by administration of phenobarbital.

This PS substance was next only to hypothalamus stimulation in the effect 


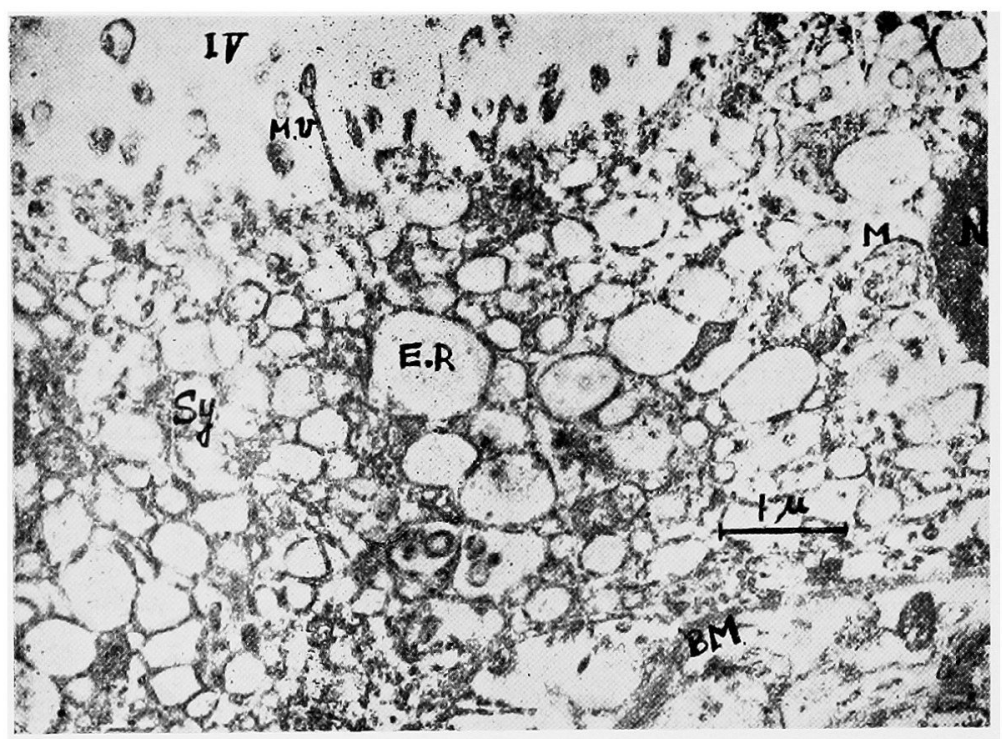

Fig. 4. Chorionic epithelium-cell of a mature placenta from acute toxemic gravida. $(\times 5,000)$

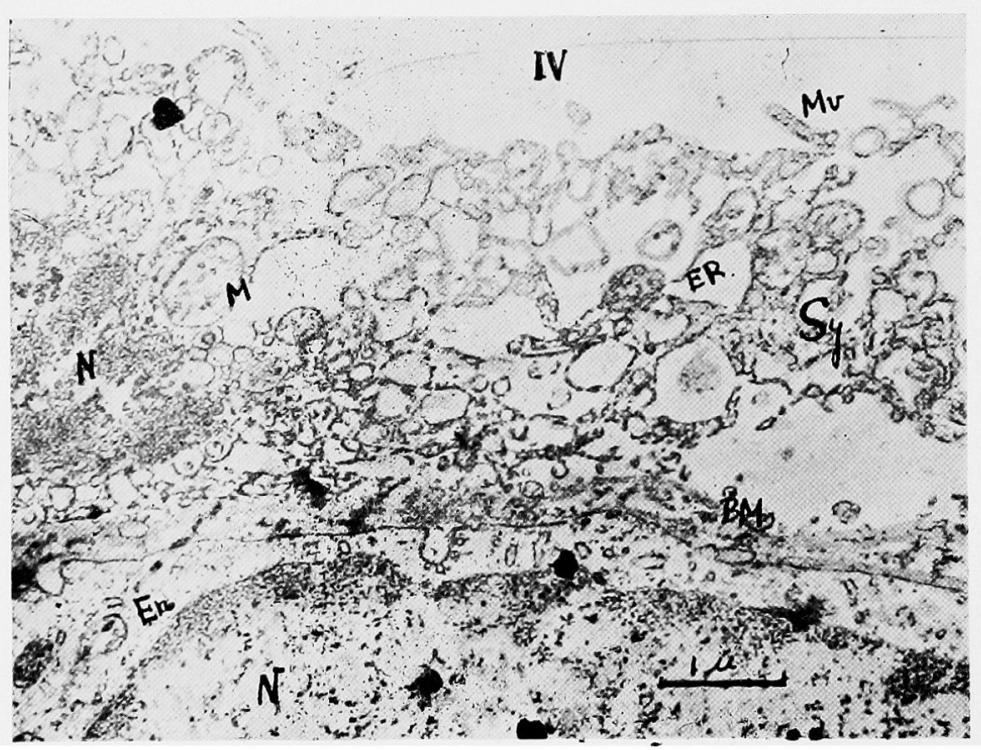

Fig. 5. Chorionic epithelium-cell in mature placenta from the patient of mixed (chronic) toxemia.

of strengthening the proneness to edema.

PS injection facilitated subsequent occurrence of Reilly's phenomenon and consequently changes resembling apoplexia uteroplacentae were sometimes 
observed.

In the PS-injected rabbits, the liver, the kidneys, the heart, the adrenal cortex and the placenta were found showing very prominent toxemia-like histological changes, but the injection could not induce eclampsia-like convulsions or pulmonary edema.

b) Kaku's substance

Pregnant rabbits were intravenously injected with $100-200 \mathrm{mg}$ of Kaku's substance (KPS). It showed as little effect of intensifying proteinuria as Magara's substance, that is, nearly none. The effect in promoting water retention was weaker than with hypothalamus stimulation or Magara's substance. The injection caused mild toxemia-like histological changes in the liver, the kidneys and the heart of the animals and the liver and the kidneys of their fetus, but no histological changes in the placenta and the adrenal cortex, no convulsion and no pulmonary edema at all.

c) Thromboplastin

Sechneider opines that thromboplastin is the cause of toxemia. Pregnant rabbits were intravenously injected with $5 \mathrm{cc}$ of $1: 200$ diluted supernatant obtained by centrifuging $0.1 \mathrm{~g}$ of thromboplastin extracted from rabbit brain with aceton and mixed with $2 \mathrm{cc}$ of physiological saline. This injection caused no toxemic symptom.

d) Tohoku substances (Co-worker: H. Yoshizaki, K. Noda and R. Hashimoto)

The underlisted 10 different substances were separately extracted from normal and toxemic placentae by the isolation method shown in Tab. II.

The effect on blood pressure and the antidiuretic action were the strongest with Comp. IV from toxemic placentae and Comp. I from normal and toxemic

\section{TABLE III. Tohoku Substances}

\begin{tabular}{|c|c|c|}
\hline \multicolumn{2}{|c|}{ Component I : } & Protein which is insoluble in water at $\mathrm{pH} 5.8$. \\
\hline Comp. & II : & Protein which is insoluble in water at $\mathrm{pH} 5.0$. \\
\hline Comp. & III : & DNA. \\
\hline Comp. & IV : & $\begin{array}{l}\text { Protein which is precipitable at } 3 / 10 \text { saturation } \\
\text { of its solution with ammonium sulfate. }\end{array}$ \\
\hline Comp. & $V:$ & $\begin{array}{l}\text { Protein which is precipitable at } 4 / 10 \text { saturation } \\
\text { of its solution with amm. sulf. }\end{array}$ \\
\hline Comp. & VI : & $\begin{array}{l}\text { Protein which is precipitable at } 5 / 10 \text { saturation } \\
\text { of its solution with amm. sulf. }\end{array}$ \\
\hline Comp. & VII : & $\begin{array}{l}\text { Protein which is precipitable at } 6 / 10 \text { saturation } \\
\text { of its solution with amm. sulf. }\end{array}$ \\
\hline Cor & VIII : & Itinsulfuric acid. \\
\hline & IX : & PNA. \\
\hline & $\mathrm{X}:$ & Crude DNP. \\
\hline
\end{tabular}


placentae. Comp. IV from toxemic placentae only showed a barely perceptible effect of aggravating proteinuria. The histological changes following injection of Comp. IV were much more intense for experimentally inducing toxemia. For the other findings, vide Tab. IV.

Table IV. Effect of Tohoku Substances in Causing Toxemic Changes

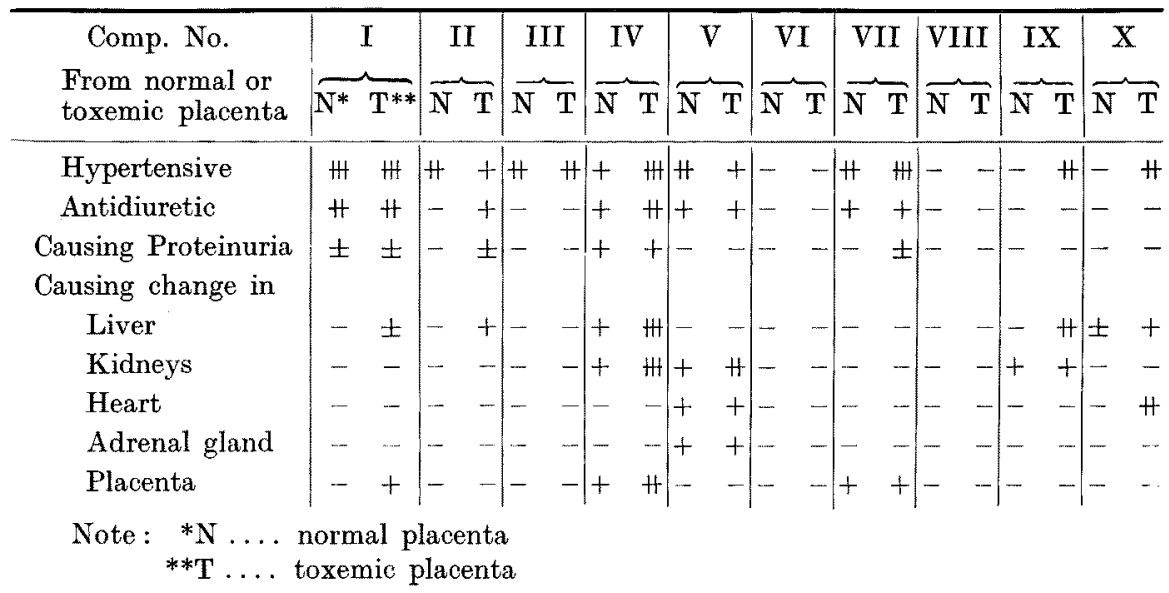

The results show that generally a component extracted from toxemic placenta has stronger effect than the same component extracted from normal placenta, except the effect on blood pressure in Comps. II and V, and that the different components exert effect on different organs.

e) Corticoids in placenta (Co-worker: T. Adachi, H. Takikawa and K. Saito)

No DOC was detected in the placenta but some other chemocorticoids could be detected by paper chromatography, as shown in Tab. V.

The anemia in toxemic cases consists mainly in hydremia mixed with slight genuine anemia. When rabbits were injected with mineral-corticoid,

TABLE V. Chemocorticoids in $\mu \mathrm{g}$ (per placenta)

\begin{tabular}{l|ccc|c}
\hline \multirow{2}{*}{ Placenta } & \multicolumn{3}{|c|}{ Glucocorticoids } & Mineralocorticoids** \\
\cline { 2 - 4 } & Tetra-h.-fr. Comp. F. Comp. E. & \\
\hline $\begin{array}{l}\text { Normal } \\
\text { (spont. delivery) }\end{array}$ & 32.2 & 8.7 & 60 & 17.5 \\
$\begin{array}{l}\text { Normal } \\
\text { (cesarean section) }\end{array}$ & 45 & 12.5 & 50 & 15 \\
$\begin{array}{l}\text { Toxemic } \\
\text { (spont. delivery) }\end{array}$ & 40 & 8.3 & 66.6 & 30
\end{tabular}

Note: * Tetra-hydro-fraction, giving spot more polar to that of Compound F. ** Giving spot between Compound B and DOC. 
gonadotrophin, cortisone (Compound E) or posterior pituitary hormone, a change resembling toxemic anemia was induced in them.

f) Pituitrin-like substance and amines from placenta (Co-worker: K. Honda and T. Kadowaki)

In the human placenta, we could demonstrate $10-18 \mathrm{mU} / \mathrm{g}$ of oxytocin-like substance. This value was somewhat higher than the content of the substance in the maternal serum. Guggisberg7) and Fellner ${ }^{8}$ have already reported on the vassopressin-like substance extracted from the placenta, and we also could comfirm the strong pituitrin-like action of it.

The content of 5-hydroxytryptamin (serotonin) in the placenta was 0.12 $\mu \mathrm{g} / \mathrm{g}$ and that in blood was $0.08 \mu \mathrm{g} / \mathrm{ml}$.

The other contents of the placenta were nor-adrenalin, guanidine, tilamin and some substances not to be identified. Substances mentioned above seemed. to cause some types of hypertonia as well as anemia.

\section{Toxemia and Corticoids}

a) Glucocorticoids (Co-worker: H. Kawahara and H. Takikawa)

The past reports claim that the urinary $17-\mathrm{OH}$-corticoid is reduced in toxemia. The results of bioassay on the subject by Kawahara confirmed this claim. When, however, Takikawa measured the urinary content of chemocorticoids in toxemia, Compound $\mathbf{F}$ and Compound $\mathbf{E}$ as well as the total 17-OH-CS were found larger than in normal pregnancy.

b) Mineralocorticoids (Co-workers: T. Adachi and H. Takikawa)

DOC was detected neither in the urine nor in the blood $(500 \mathrm{cc})$ of toxemic patients. A mineralocorticoid forming a spot between those of DOC and Compound B in paper chromatography (Bush C system) was found increased in toxemia., increasing and decreasing parallel with the grade of the trouble. Aldosterone was found increased in the gravidae with severe edema.

\section{Toxemia and Renin}

Y. Kikuchi, one of the co-workers, extracted renin by an improved method devised by judicious combination of the processes of Yoshida ${ }^{91}$ and Landis ${ }^{10}$. He then measured the renin content in serum of 20 toxemic patients and proved its existence in 10 cases among the hypertensive toxemias. A renin-like substance was isolated from the placenta and the lung of animals in which anoxia had. been induced.

Of the types of toxemia, the mixed type (the authors are used to call it "chronic toxemia") represents the disorder with long-persistent toxemic symptoms after delivery. These persistent symptoms consist in most part of hypertension 
or hypertension plus proteinuria and but rarely of proteinuria alone. Thus, chronic toxemia nearly always shows the symptom of hypertension and accompanies sclerotic changes in the small blood vessels.

In the above-described experimental toxemia, we could not induce disturbance resembling chronic toxemia. Masson et al11-12) report that injection of renin plus DOC or renin puls glucocorticoid induces vascular sclerosis in animals. Our follow-up experiments brought about similar results: When rats were injected subcutaneously with hog renin, in combination with $3 \mathrm{mg}$ of DOC, with $1.5 \mathrm{mg}$ of cortisone or $1.5 \mathrm{mg}$ of hydrocortisone, over 14 days in succession, sclerosis was induced in the renal blood vessels. The same result was obtained when the renin-like substance extracted from the placenta was applied in the same manner instead of hog renin.

These results suggest that chronic toxemia is induced when renin or the reninlike substance from the placenta comes into reaction with the mineralo- and gluco-corticoids serected from the adrenal glands and the placenta over considerable duration. In human cases also, it has been observed that chronic toxemia is apt to occur when toxemic hypertension has persisted for more than 2 weeks during pregnancy.

\section{COMMENT}

Late pregnancy toxemia is a syndrom. It would be more convenient for us if the genetic factor of this syndrome is single, but if we start to search for such a single cause from the outset, it may lead to erroneous conclusions. It seems, however, very probable that one principal cause is responsible for the major parts of the symptoms.

After many years of research work and observation of many toxemia cases, we have been led to infer that the cause and the mechanism of pathogenesis of toxemia are something as summarized in the following:

When the influence of the physiological phenomenon of pregnancy goes beyond the capacity of the pregnant to stand, a disturbance occurs in the function of the hypothalamus. This disturbance is probably occasioned by stimulated neurosecretion and placental products, and amplified by emotional uneasiness etc. The dysfunction of the hypothalamus, besides causing proteinuria, edema, hypertension, eclampsia, preparatory tendency to pulmonary edema, change of the eye fundus and toxemic histological changes in the liver, the kidneys, the heart, the adrenal glands and the fetus, also causes change in the nature of the blood in the intervillous spaces, to form deposits of fibrin on the villi which in turn entails anoxia of the fetal placenta, thus completing the specific changes of toxemic placenta. Many kinds of protein are then released from the placenta, which causes edema, hypertension, abruptio placentae, toxemic anemia, and toxemic changes 
in the maternal liver, adrenal glands, heart and kidneys and in the fetus. Different kinds of protein thus released cause troubles in different organs. The proteins released from the placenta constitute noxa also to the placenta itself and further aggravate the toxemia. Amines are also released from the placenta and beget hypertension.

The adrenocortical hormones, besides causing edema and toxemic anemia, cause chronic (mixed) toxemia by coming into reaction with renin or the reninlike substance produced in the placenta.

Chronic toxemia induces occurrence of abruptio placentae and eclamptic cerebral hemorrhage in multipara. The Reilly's phenomena due to release of adrenalin seem to play some part in the occurrence of toxemic pulmonary edema and abruptio placentae. The above process is schematized in Fig. 6.

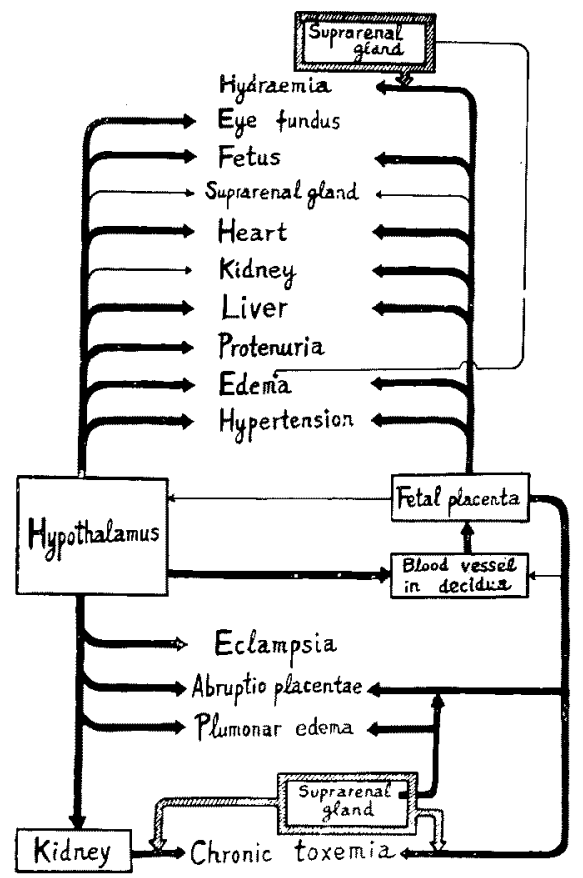

Fig. 6. Mechanism of Pathogenesis of Late Pregnancy Toxemia.

\section{CONCIUUSION}

Basing ourselves on the results of a long series of experiments, the present author has made inferences on the probable genesis of late pregnancy toxemia as summarized as follows:

1) If the influence of normal pregnancy gets beyond the capacity of the pregnant to withstand, a disturbance is induced in the function of her hypothalamus. 
2). The dysfunction of the bypothalamus directly causes edema, proteinuria, hypertension, eclampsia, retinal changes, histological changes in some maternal as well as fetal organs, and toxemic changes in the fetal placenta. Many kinds of protein are released from the affected placenta.

3) These proteins from the placenta in turn cause edema, hypertension, histological changes in some organs and the fetus, as well as anomalies in the placenta itself. Besides, the placenta secretes amines effective in causing hypertension and mineralo- and gluco-corticoids causing toxemic anemia.

4) The hormones secreted by the adrenal glands, in reaction with the corticoids from the placenta, cause edema and hydremia (toxic anemia).

5) Renin and the renin-like substance released from the placenta, when coming in reaction over long duration with corticoids, cause chronic or mixed toxemia.

6) The Reilly's phenomenon caused by release of adrenalin is responsible for the occurrence of toxemic pulmonary edema and abruptio placentae.

7) Thus, toxemia is a syndrome of which the dysfunction of the hypothalamus and the placental substances released thereby is the primary cause and the adrenocortical hormones, renin, adrenalin and neurohypophyseal hormones play the part of secondary causes.

8) From the above findings, we may conclude that late pregnancy toxemia may not be called "a genuine toxemia" but should be called "a dysadaptation syndrome of pregnancy".

\section{References}

1) Kurotsu, T., No-Kenkyu (Jap.), 1949, 3, 39.

2) Schneider, C.L., Amer. J. Physiol. 1947, 149, 123.

3) Magara, M., J. Japan. Obst. \& Gyn. Soc., 1959, 6, 114.

4) Kaku, M., J. Obstetr. Gynaecol. Brit. Emp., 1953, 60, 148.

5) Yoshizaki, H., Tohoku J. Exp. Med., 1957, 66, 333.

6) Couvelaire, A., Ann. Gynec. et Obst., 1912, 9, 486.

7) Guggisberg, H., Msch. f. Geb. u. Gyn., 1921, 54, 277.

8) Fellner, C., Arch. f. Gyn., 1927, 137, 1.

9) Yoshida, M., Fukuoka Igaku Zasshi (Jap.), 1940, 33, 1258.

10) Landis, E.M., Am. J. Med. Sci., 1941, $202,14$.

11) Masson, G.M.C. et al., Arch. Path., 1952, 53, 217.

12) Masson, G.M.C. et al., Am. J. Med. Sci., 1953, 226, 296.

13) Masson, G.M.C. et al., Arch. Path., 1953, 56, 23. 